\title{
From BIM to GIS at the Smithsonian Institution
}

\author{
Detlef Günther-Diringer ${ }^{\mathrm{a}}$ \\ ${ }^{a}$ University of Applied Sciences, Karlsruhe, Germany; Smithsonian Fellow March-August 2016; detlef.guenther-diringer@hs- \\ karlsruhe.de
}

\begin{abstract}
BIM-files (Building Information Models) are in modern architecture and building management a basic prerequisite for successful creation of construction engineering projects. At the facilities department of the Smithsonian Institution more than six hundred buildings were maintained. All facilities were digital available in an ESRI ArcGISenvironment with connection to the database information about single rooms with the usage and further maintenance information. These data are organization wide available by an intranet viewer, but only in a two-dimensional representation. Goal of the carried out project was the development of a workflow from available BIM-models to the given GIS-structure. The test-environment were the BIM-models of the buildings of the Smithsonian museums along the Washington Mall. Based on new software editions of Autodesk Revit, FME and ArcGIS Pro the workflow from BIM to the GIS-data structure of the Smithsonian was successfully developed and may be applied for the setup of the future 3D intranet viewer.
\end{abstract}

Keywords: BIM (Building Information Modeling), 3D-Visualization, GIS

\section{Introduction}

BIM-files (Building Information Models) are in modern architecture and building management a basic prerequisite for successful creation of construction engineering projects. Very detailed, 3D-data will be generated during the BIM-process and could be integrated in GIS-data structure. However, the compatibility of both worlds is loaded with a lot of questions and problems (Liu et al. 2017; Kolbe et al. 2011).

At the facilities department of the Smithsonian Institution more than six hundred buildings were maintained. All facilities were digital available in an ESRI ArcGISenvironment with connection to the database information about single rooms with the usage and further maintenance information. These data are organisation wide available by an intranet viewer, but only in a twodimensional representation.

Independent of the two-dimensional GIS dataset at the Smithsonian there are many activities to use very detailed BIM-files of their buildings for different facility tasks and visualizations (Kendra 2017)

During his time as a Smithsonian Fellow in 2016 a workflow was developed by the author to integrate the given BIM-models of the Smithsonian Museums along the Washington Mall into a GIS-System. The workflowdevelopment based on one hand of the experience of a large 3D City model creation process (Guenther-Diringer 2016), where five historical time steps of the German city of Karlsruhe were created, on the other hand, new possibilities through new software-editions were applied, like Autodesk Revit, FME, or ArcGISPro.

\section{Background}

On the ICC Conference 2013 in Dresden, Germany, a poster called "3D-Citymodel: 300 years Karlsruhe: 1715
$-1834-2015$ " was presented by the author. This project with extended digital 3D citymodels of different time steps (1739 - 1834 - 1915 - 1945 und 2015) was finalized at the 300 year anniversary of Karlsruhe and installed as a self-running application at the Karlsruher city museum in 2016. The technical aspects of this project, which lasts over 4 years were published at the German Cartographic News (Günther-Diringer 2016).

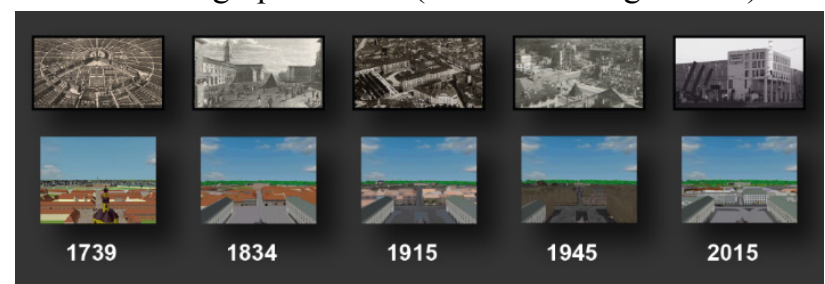

Fig. 1. Historical City Model of Karlsruhe (Source: GüntherDiringer 2016).

During this project a workflow from 2D geospatial base data to extended high-end 3D city models was developed, see Fig. 2. 


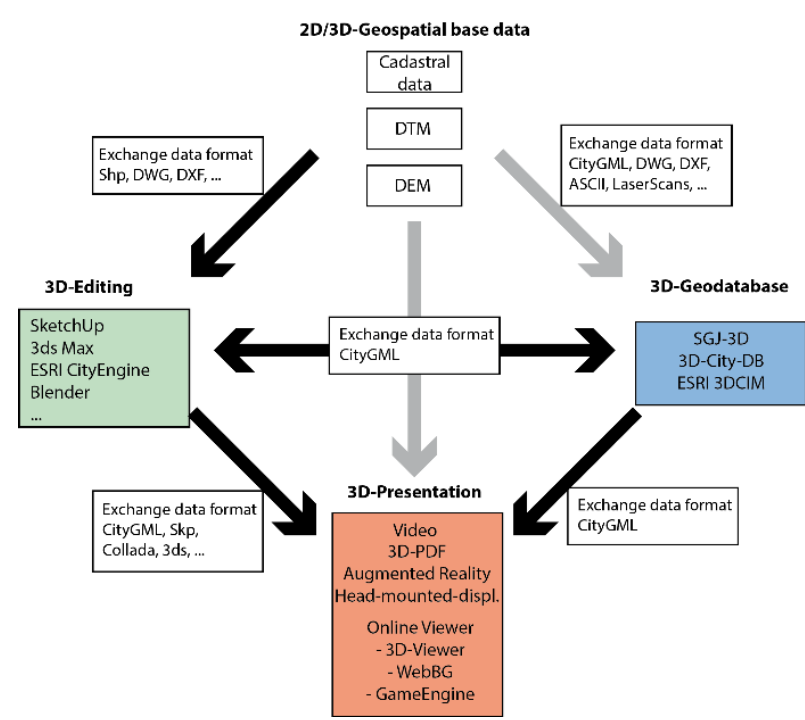

Fig. 2. Workflow of the historical city model of Karlsruhe (Source: Günther-Diringer 2016).

\section{Project workflow}

\subsection{BIM models of the Smithsonian Institution}

Goal of the carried out project was the development of a workflow from available BIM-models to the given GISstructure. The test-environment were the BIM-models of the buildings of the Smithsonian museums along the Washington Mall. The BIM-models were available with a file size from $25 \mathrm{MB}$ (Smithsonian Castle) up to $1 \mathrm{~GB}$ (National Museum of African American History and Culture) in the Autodesk Revit-file format, a typical BIM application.

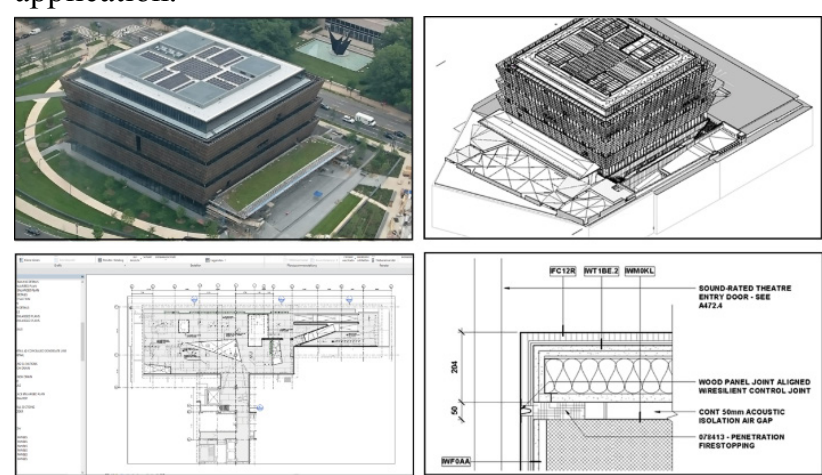

Fig. 3. National Museum of African American History and Culture: photo and BIM-file in different detail views (Source: Günther-Diringer, Smithsonian Institution).

\subsection{Available software configuration}

To integrate the BIM models into GIS the mentioned workflow (fig. 2) had to be modified. The input BIM-data were available as Autodesk Revit-files. Autodesk Revit is a standard software for BIM-Modeling (Autodesk 2017). On the GIS-part of the project next to the ESRI ArcGISDesktop-Software the new ArcGIS Pro 1.3 was used (Esri 2017). With the new 3D capabilities of ArcGIS Pro it was an appropriate target GIS-System for the available BIM-models. With the integration of ArcGIS Pro the workflow has to be adapted too, because of the now available, integrated 3D capabilities, many of the necessary $3 \mathrm{D}$ tasks of $3 \mathrm{D}$ city modelling can now carried out in the same GI-environment as the 2D geodata editing.

However, due to the file size and the complexity of the single BIM-files it is not useful to import the full BIMmodel for example by the fbx-format into the GIS. In comparison to an available open data set of 50.000 buildings of Washington DC (DC.gov 2017: dcatlas.dcgis.dc.gov/download/bldgPly_3D.zip) with about $95 \mathrm{MB}$, a single BIM-model were up to $1 \mathrm{~GB}$. The BIM-data has to be restructured and reduced, but without the loss of the internal topological structure and the necessary connections to the database information. For this task, the FME (Feature Manipulation Engine) of Safe Software was used (Safe 2017). FME is an ETL-Software (Extract, Transform, Load) which supports more than 400 different data formats and has extended transformation functionalities by more than 465 transformers.

\subsection{Applied transformation workflow}

After installation of FME on a computer where Autodesk Revit was installed as well, a FME-Export-plug-in at Autodesk Revit was available and due to diverse setting options the file size of the original Revit file could be reduced, f.e. from $25 \mathrm{MB}$ to $7 \mathrm{MB}$.

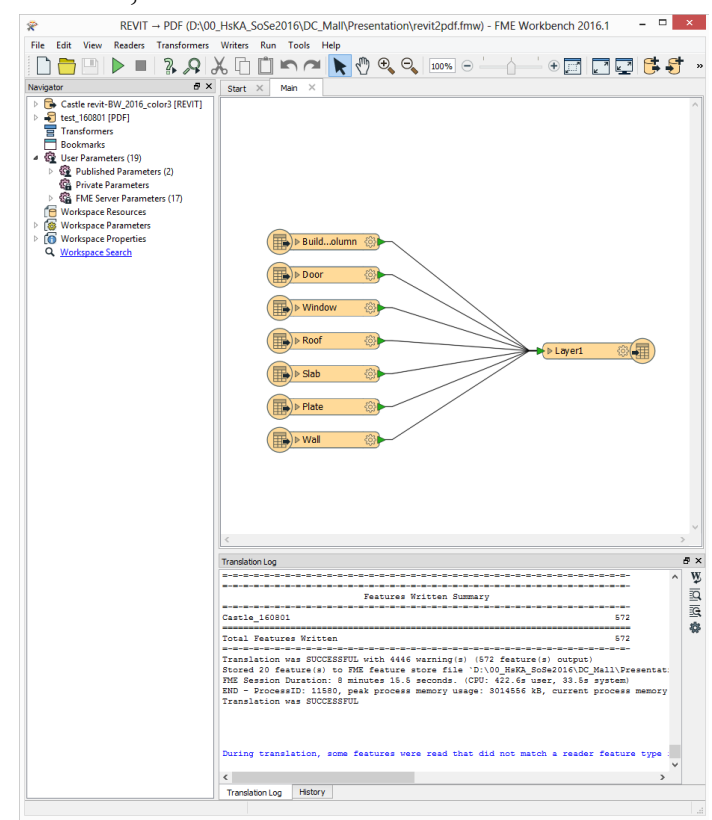

Fig. 4. Example-workflow in FME.

With further processing inside FME export to Adobe 3Dpdf or ArcGIS file geodatabase-format (.gdb) was possible. The file size was further reduced to $2 \mathrm{MB}$ (pdf), respective $4 \mathrm{MB}(\mathrm{gdb})$.

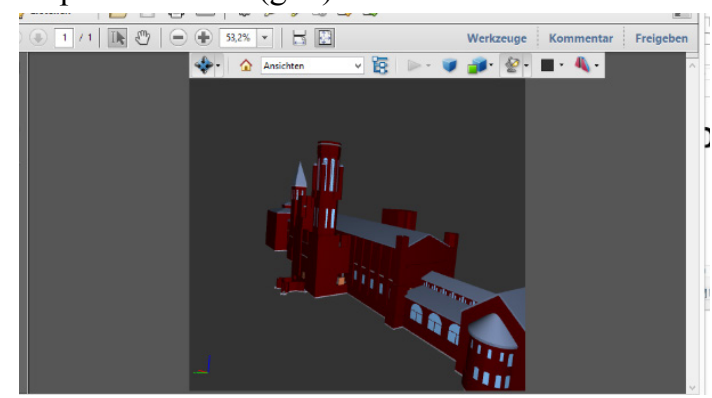


Fig. 5. BIM-model of Smithsonian castle in Adobe 3D pdf.

BIM models usually did not have geocoding information. Normally the origin of their coordinate system, the project base point, is in the center of the building. However, this problem could be solved with FME as well. The co-ordinates and the geodetic datum of the appropriate building have to be available (at least on point at a building corner). In Revit the available survey point, by default identical with the project base point, has to be shifted to the building corner, where the coordinates are known. Inside FME-Software the transformer "offsetter" had trans-formed the building coordinates to the appropriate shift. Later in ArcGIS the correct geodetic datum has to be chosen and the imported model was placed at its correct position.

A whole pipeline of transformers can be set up with an appropriate file export format, f.e. Adobe 3D-pdf or ArcGIS file gdb. At the end of the process, the file size was reduced to $1 / 6$ of the origin and can be imported to ArcGIS Pro as a multipatch-object for $3 \mathrm{D}$ visualization or as a polygon-object for combined $2 \mathrm{D} / 3 \mathrm{D}$ visualization, if the single rooms of one storey would be of interest (fig. 6 and fig. 7).

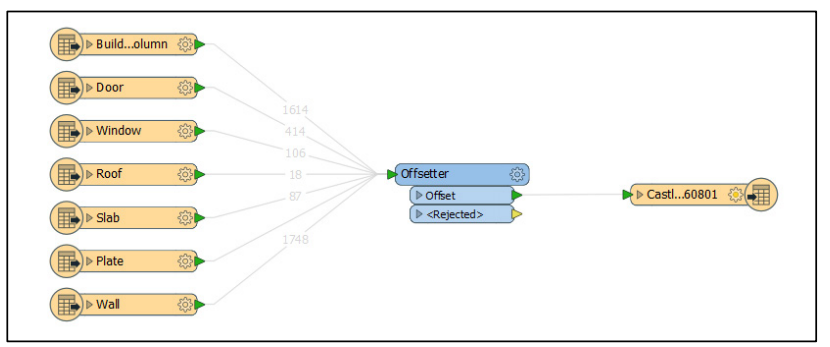

Fig. 6. FME-transformation from Revit-export with geocoding offset to a 3D multipatch object in a ArcGIS file gdb.

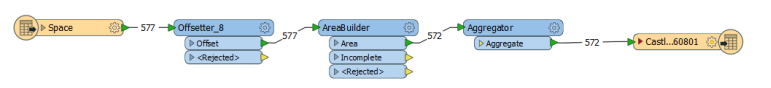

Fig. 7. FME-transformation from Revit-export with geocoding offset and separation by different storeys to a $2 \mathrm{D}$ polygon object in a ArcGIS file gdb.

\subsection{ArcGIS Pro integration}

Due to the FME transformation process, the generated geodatabase-files could be loaded into ArcGIS Desktop as well as to ArcGISPro 1.3 and combined with other georelated data.

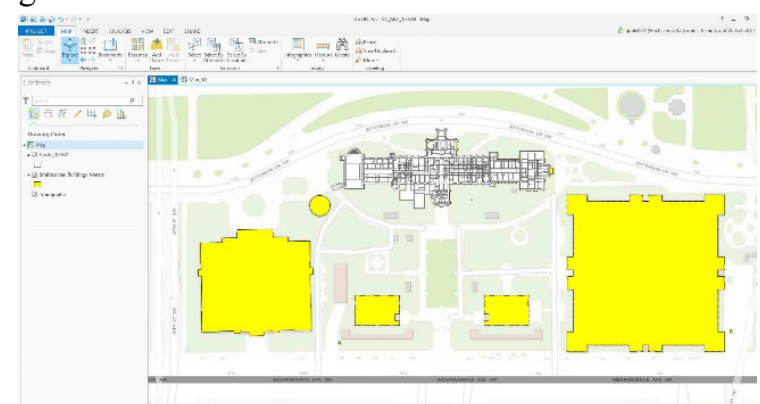

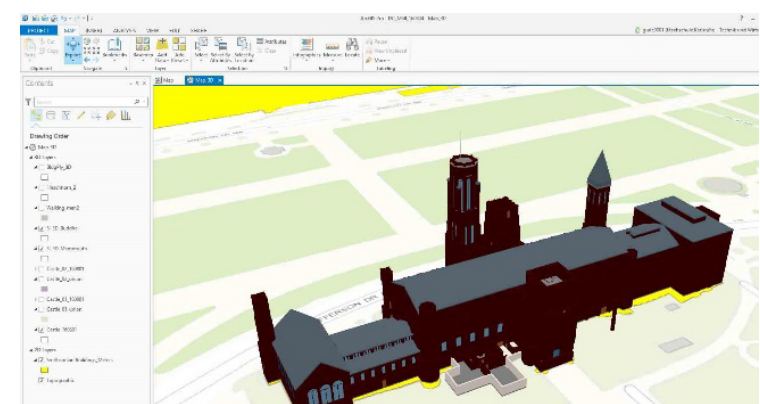

Fig. 8. ArcGIS Pro with imported multiptach file, 2D view (left) and $3 \mathrm{D}$ view (right).

The imported 2D polygon file, which was created based on FME workflow in Fig. 7, could be displayed separated by the different floors, with the single rooms as an important base geometry. Due to the preserved IDs the single geometry could be linked to the available facility database, with the necessary information about usage, maintenance and other important information.

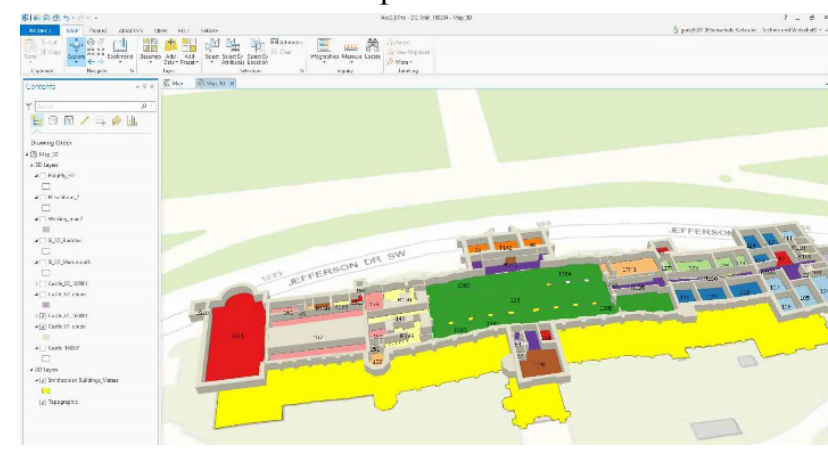

Fig. 9. ArcGIS Pro with imported polygon file of single floor with visible IDs of the single rooms.

With the integration of the BIM-models into the ArcGIS Pro environment, all of the available GIS-functionality could be carried out in combination with the BIM data. In Fig. 10 is an example of a combination of the geocoded BIM data with results of the very sophisticated Smithsonian X 3D-project, "Smithsonian X 3D launches a set of use cases which apply various 3D capture methods to iconic collection objects, as well as scientific missions" (Smith-sonian Institution 2017). The downloadable 3D scan "Woolly Mammouth" (3d.si.edu/downloads/55) was import-ed, geocoded and placed in one of the rooms of the Smithsonian Castle, together with an available "Cosmic Bud-dha" statue.

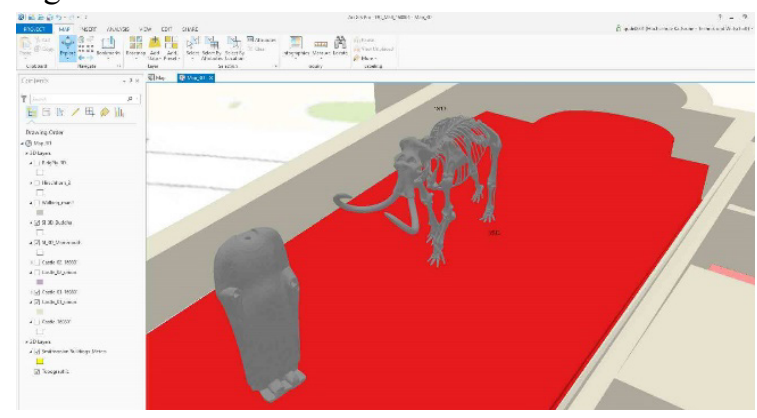

Fig. 10. ArcGIS Pro view with a single room and combined 3D scanned objects.

Since a few years the Woolly Mammouth is not available in one of the Smithsonian exhibitions. In combination 
with single scanned 3D-objects, available BIM-room structure and the given georeferencing of the objects a digital preservation of former exhibitions could be achieved as a future application.

Another advantage of the integration into GIS structure is the combination with other georelated data. Together with an open data set of 50.000 buildings of Washington DC, mentioned in chapter 2.2, the imported BIM-data gave an excellent view for further planning applications (Fig. 11).

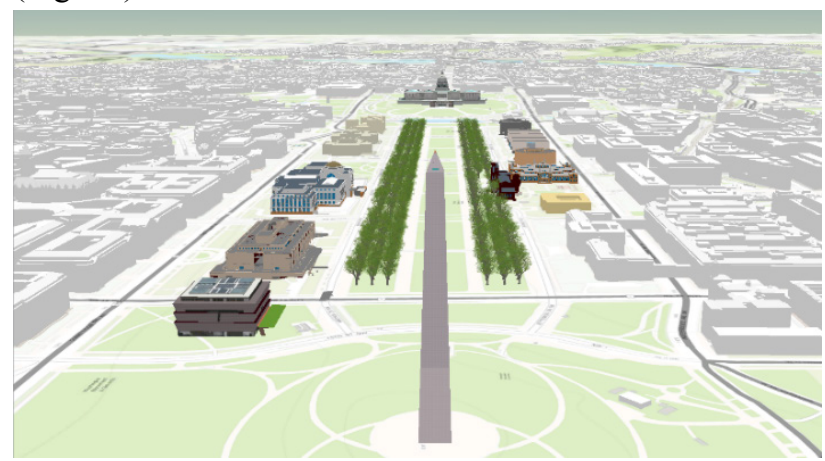

Fig. 11. BIM-models of the Washington Mall combined with open geodata.

For producing of high-end animation videos, it was not necessary to leave the GIS environment. Due to new extended animation possibilities in ArcGIS Pro, a lot of functionality was now available, with the decisive advantage that the voluminous geodata could be processed in the same system and did not have to be exported to another software package for 3D construction and video processing. In Fig 12 a few screenshots of a video which shows the historical development of the mall from 1791, before the founding of Washington DC up to the new, in 2016 opened Museum of African American History and Culture.
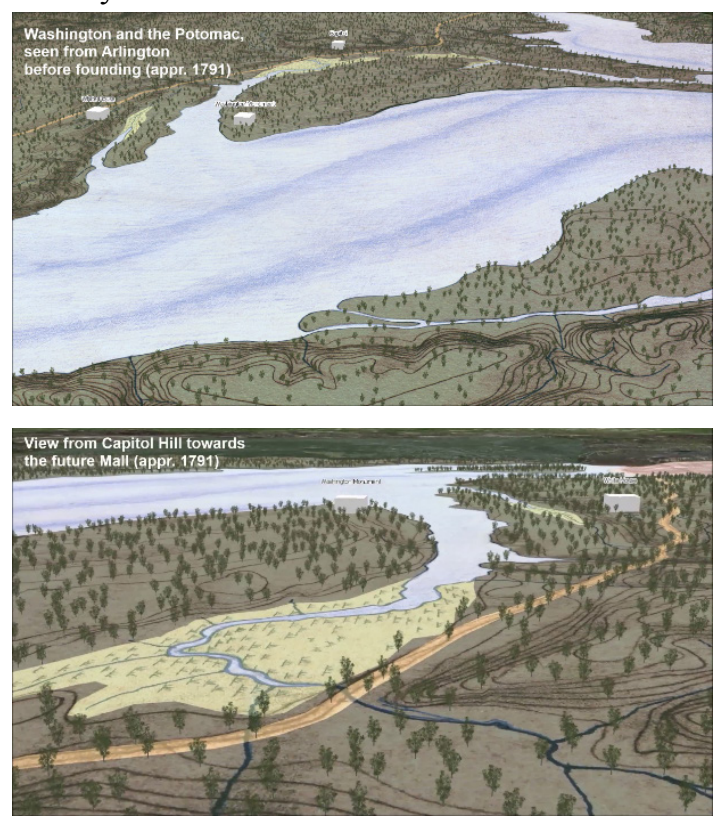
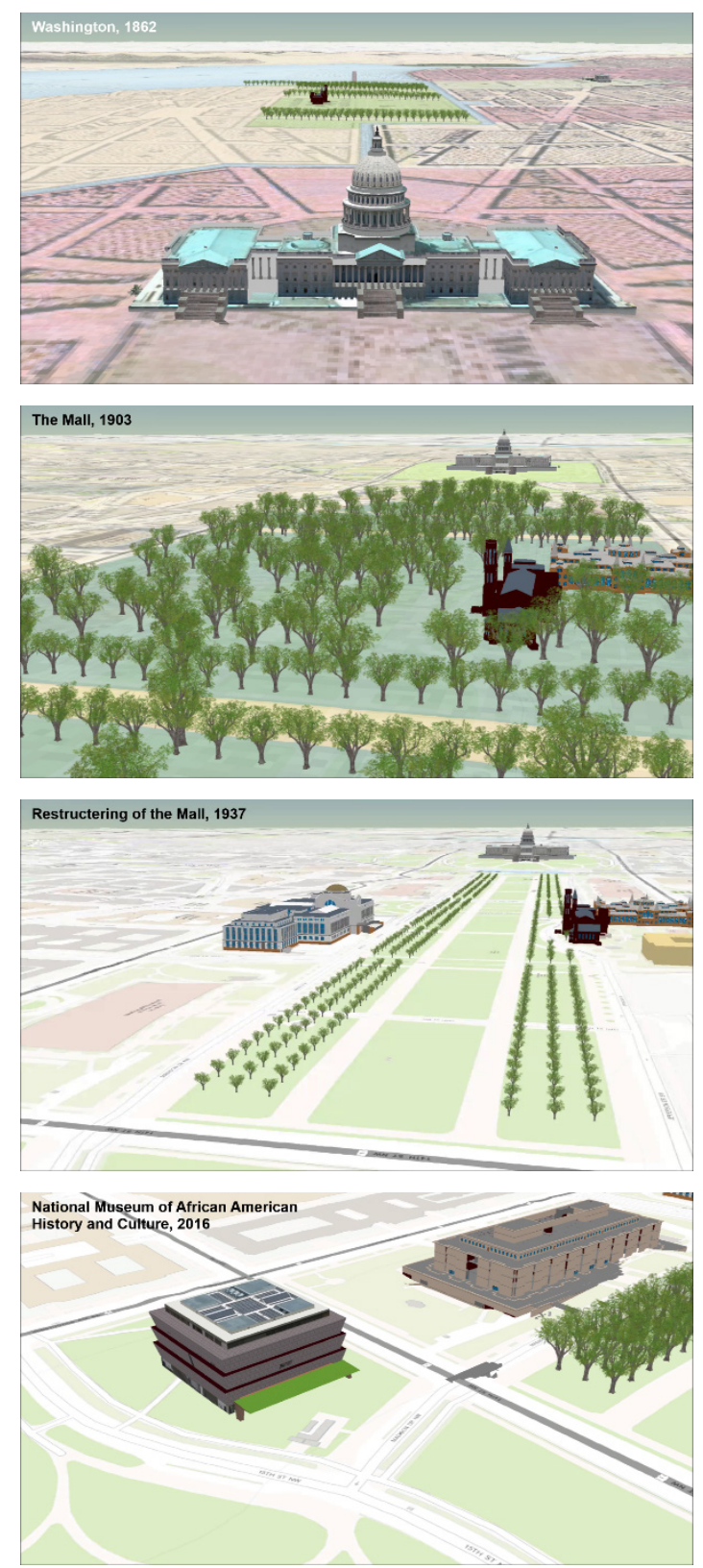

Fig. 12. Screenshots of the video generated by ArcGIS Pro animation functionality.

\section{Conclusions}

The workflow from BIM to the GIS-data structure of the Smithsonian was successfully developed and may be applied for the setup of the future 3D intranet viewer. There is the possibility to integrate CityGML as a xmlbased standard data format for 3D citymodels (OGC 2017) into the workflow. The FME-software offers sample work-flows from BIM to GIS based on the CityGML-format. But this format was not in use at the Smithsonian facility up to now and therefore it was not applied in the described workflow.

Next to the technical aspect, the future application of the workflow is very much based on organizational issues. Which kind of facility data should be held in which level of detail to support which tasks and to serve which user group inside and outside the Smithsonian Institution? A lot of questions has to be answered, before the time 
consuming process to set up the possible future $3 \mathrm{D}$ intranet viewer can be started. But during this process different departments has to communicate to each other. The 3D realistic view of their common data will give them all better possibilities for solving their complex future tasks.

\section{Acknowledgements}

I am grateful to Dan Cole, GIS-Coordinator at the Smithsonian Institution, for organizing my research term as a Smithsonian Fellow from March - August 2016 at the National Museum of Natural History in Washington DC.

\section{References}

Autodesk (2017). Autodesk Revit. www.autodesk.com/revit. Accessed 28 Feb 2017.

DC.gov (2017). Open dataset of Washington buildings. dcatlas.dcgis.dc.gov/download/bldgPly_3D.zip. Accessed 28 Feb 2017.

ESRI (2017). ArcGIS Pro. www.esri.com/arcgispro. Accessed 28 Feb 2017.

Günther-Diringer, D. (2016). Historisches 3DStadtmodell von Karlsruhe. Kartographische Nachrichten 66 (2).

Kendra, S. (2017): Developing a 3D Building Information Management Practice. Smithsonian Institution. Presentation held on 5th quadrennial GIS @ the Smithsonian conference (unpublished).

Kolbe, T. H.; König, G.; Nagel, C. (2011). Advances in 3D Geo-Information Sciences: Springer Berlin Heidelberg. Online available https://books.google.de/books?id=hqf5ylwRhdIC. Accessed 28 Feb 2017.

Liu, Xin; Wang, Xiangyu; Wright, Graeme; Cheng, Jack; Li, Xiao; Liu, Rui (2017). A State-of-the-Art Review on the Integration of Building Information Modeling (BIM) and Geographic Information System (GIS). In: IJGI 6 (2), S. 53. DOI: 10.3390/ijgi6020053.

OGC (2017). CityGML-standard. www.opengeospatial.org/standards/citygml. Accessed 28 Feb 2017.

Safe (2017): FME Feature manipulation Engine. www.safe.com. Accessed 28 Feb 2017.

Smithsonian Institution (2017). Smithsonian X 3D. www.3d.si.edu. Accessed 28 Feb 2017. 